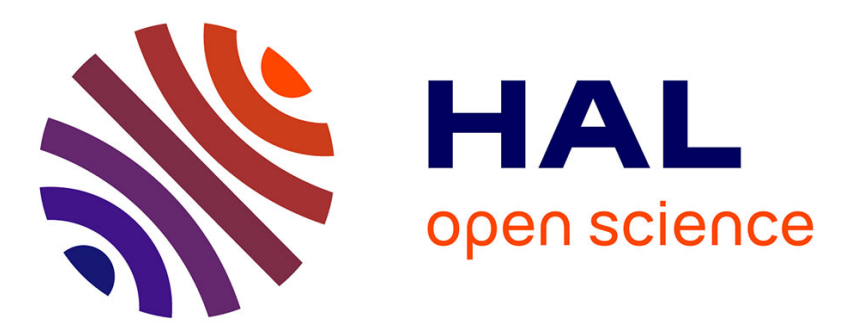

\title{
The influence of irradiation induced vacancies on the mobility of helium in boron carbide
}

Kevin Gillet, Guido Roma, Jean-Paul Crocombette, Dominique Gosset

\section{To cite this version:}

Kevin Gillet, Guido Roma, Jean-Paul Crocombette, Dominique Gosset. The influence of irradiation induced vacancies on the mobility of helium in boron carbide. Journal of Nuclear Materials, 2018, 512, pp.288-296. 10.1016/j.jnucmat.2018.10.020 • cea-02339428

\section{HAL Id: cea-02339428 https://hal-cea.archives-ouvertes.fr/cea-02339428}

Submitted on 20 Nov 2019

HAL is a multi-disciplinary open access archive for the deposit and dissemination of scientific research documents, whether they are published or not. The documents may come from teaching and research institutions in France or abroad, or from public or private research centers.
L'archive ouverte pluridisciplinaire HAL, est destinée au dépôt et à la diffusion de documents scientifiques de niveau recherche, publiés ou non, émanant des établissements d'enseignement et de recherche français ou étrangers, des laboratoires publics ou privés. 


\title{
The influence of irradiation induced vacancies on the mobility of helium in boron carbide
}

\author{
Kevin Gillet ${ }^{1}$, Guido Roma ${ }^{1}$, Jean-Paul Crocombette ${ }^{1}$, Dominique Gosset ${ }^{2}$ \\ ${ }^{1}$ DEN-Service de Recherches de Métallurgie Physique, CEA, Université Paris-Saclay, \\ F-91191 Gif sur Yvette, France ${ }^{2}$ DEN-Service de Recherches de Métallurgie Appliquée, \\ CEA, Université Paris-Saclay, F-91191 Gif sur Yvette, France
}

\begin{abstract}
Boron carbide, used as a neutron absorber, undergoes nuclear reactions producing relevant quantities of He. The understanding of He kinetics at the atomic scale in the material is still in its infancy, in spite of decades of experimental work devoted to the characterization of He containing, irradiated, boron carbide samples. The interplay of He itself with intrinsic defects created by irradiation on kinetics is still almost completely unknown.

In this paper we present an exhaustive study of vacancies and substitutional helium impurities in boron carbide using Density Functional Theory. Analyzing the stability and mobility of these defects allows us to consider diffusion mechanisms other than the known interstitial mechanisms.

We find that vacancies trap He interstitials, raising the activation energy of $2 \mathrm{D}$ diffusion to approximately $2 \mathrm{eV}$. The trapping mechanism is different according to the charge state of the vacancy : in $p$-type conditions, when vacancies are neutral or positive, He diffuses via a dissociative mechanisms and is trapped in a substitutional position; in $n$-type conditions, negative vacancies trap He atoms traveling in an adjacent $\{111\}$ plane by a charge transfer driven distorsion.

No favorable vacancy assisted diffusion mechanism was identified for substitutional He atoms, except the dissociative one previously mentioned. Other possible vacancy diffusion mechanisms, which we also analyzed, are hindered by the high activation energy of vacancy self-diffusion.
\end{abstract}

Keywords: Density functional theory, Boron carbide, Helium, Vacancies, Kinetics, Nudged elastic bands 


\section{Introduction}

Boron carbide, due to the very high neutron absorption cross-section of the boron isotope ${ }^{10} \mathrm{~B}$, and fairly smooth dependence of it on neutron energy, has been extensively employed as neutron absorber in the nuclear industry for several decades, including for fast breeder reactors[1, 2].

It constitutes the core of the control rods, placed among the fuel rods in the reactor, which are meant to regulate the rate of fuel fission or to shutdown the reactor. It is crucial to maintain the integrity of control rods for security and operational management, but due to the degradation of irradiated boron carbide, a brittle material, their lifetime, which is around 10 to 15 years in thermal neutron reactors, drops to less than 5 years in fast neutron reactors [2]. Being at the core of the nuclear power plant, the replacement of control rods is expensive and has to be carried out when the reactor is shut down. Therefore, optimizing boron carbide behavior under irradiation is one of the key features of next generation nuclear power plants viability.

Boron carbide is also increasingly widespread among other important technological applications, in particular related to its hardness - one of the highest known - , low density and good chemical stability, like for example armor plating [3, 4], processing tools, composites fibers and more. As a semiconductor, it could perhaps be envisaged for functional applications; however the wide stoichiometry range and still not fully understood atomic disorder has, up to now, prevented such applications.

In spite of the technological importance of the material both self- and impurity diffusion, which are important in processing, microstructure control, and irradiation behavior, are very scarcely understood in this material.

In particular, the interplay of helium and the point defects created by neutron irradiation, and their mobility, is crucial in order to understand and control the formation of helium bubbles, which ultimately control internal strain[5] and the lifetime of control rods[2].

Boron carbide, somewhat improperly often referred to as $\mathrm{B}_{4} \mathrm{C}$, occurs in variable stoichiometry. In order to explain these features various defective structures were investigated, in particular chain vacancies [6, 7], antisites[8, 9] and, very recently, boron interstitials[10]. The goal was, however, mainly phase stability and not the properties of isolated defects, nor their role in mass transport kinetics. In spite of a number of experimental works devoted to helium bubbles[11, 12, 13, 14, 15, 16, 17, 18, 19, 20, 21] and the related 
question of helium kinetics[20, 22, 23, 24], not much is known on self-diffusion.

In a previous theoretical work we showed how helium interstitial $2 \mathrm{D}$ diffusion could bias the shape of helium bubbles[25]. A subsequent theoretical study[26] has confirmed our results for He and investigated the mobility of lithium, the other product of the nuclear reaction triggered by ${ }^{10} \mathrm{~B}$ neutron absorption. Although some results on neutral vacancies where presented in these two works, no attempt was made to assess the influence of such defects on the mobility of He.

In order to gain a deeper understanding of He bubble formation under irradiation conditions it is necessary to assess the role of intrinsic defects created by irradiation and, in particular, the role of vacancies, which constitute possible bubble nucleation sprouts.

In this work we focus on the influence of intrinsic vacancies created under irradiation on the diffusion of helium, which is produced by nuclear reactions in boron carbide submitted to a neutron flux. Such a picture can also be simulated by helium implantation[20], possibly coupled with ion irradiation. We investigate the possible mechanisms at the atomic scale by means of first principles calculations in the framework of Density Functional Theory (DFT).

The paper is organised as follows : in section 2 we discuss methodological aspects of our calculations. The results section 3 is splitted in two subsections: the first, 3.1, devoted to vacancy formation and migration, the second, 3.2 to substitutional helium and its migration mechanisms involving vacancies. We conclude with a discussion on the possible contribution of those mechanisms to He diffusion (section 4).

\section{Computational Details}

The results presented in this paper rest upon first-principles calculations as formulated in the Density Functional Theory (DFT) framework. Defect stability and defect mobility are respectively quantified in term of formation energy and migration energy obtained through total energy calculations of several configurations.

Boron carbide rhombohedral phase exists over a large range of stoichiometry: the literature reports boron carbides from 8.6 to 18.8 at.\% $\mathrm{C}[27,28]$ even if precise boundaries are still under debates [29]. These stoichiometry changes in boron carbide are thought to be accomodated thanks to local

disorder in the structure [30]. Thus model structures of boron carbide are 


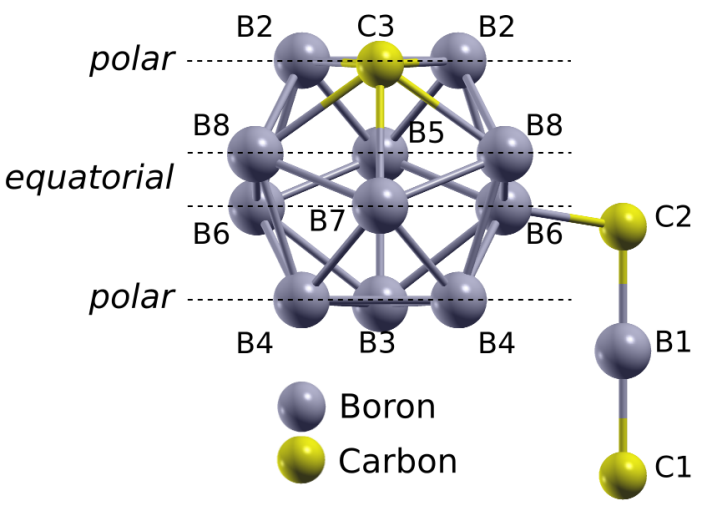

Figure 1: The $\mathrm{B}_{11} \mathrm{C}^{\mathrm{p}} \mathrm{CBC}$ structure, atoms are divided in 4 groups : polar (C3, B2, B3, $\mathrm{B} 4)$, equatorial (B5, B6, B7, B8), chain end (C1, C2) and chain center (B1).

necessarily approximations. In this study we chose to represent the boron carbide bulk crystal by the carbon-rich crystallographic structure $\mathrm{B}_{11} \mathrm{C}^{\mathrm{p}} \mathrm{CBC}$ $\left(\mathrm{C}^{p} \equiv\right.$ carbon in polar position) which is considered as the most stable theoretical structure over the whole homogeneity range at $0 \mathrm{~K}[31,32,33]$. This structure is described by a 15-atom elementary cell of rhombohedral symmetry with an icosahedron $\mathrm{B}_{11} \mathrm{C}$ at each vertex and a chain $\mathrm{C}-\mathrm{B}-\mathrm{C}$ on the main diagonal ( $\langle 111\rangle$ direction). The 12 icosahedron sites are divided in two distinct types resulting from their chemical environment : the 6 equatorial sites which are bonded to the chains (B5, B6, B7, B8) and the 6 polar sites which are directly bonded to other icosahedra (C3, B2, B3, B4) ; the chain sites correspond to the carbon extremities $(\mathrm{C} 1, \mathrm{C} 2)$ called chain end and the boron chain center B1 (Fig. 1).

Boron carbide is experimentally observed of rhombohedral symmetry [34], and carbon atoms on the icosahedra are supposed to be found randomly distributed between the six crystallographically equivalent polar sites. Thus the use of periodic boundary conditions leads to an unphysical periodic arrangment of the carbons on the icosahedra which breaks the rhombohedral symmetry into monoclinic symmetry. The resulting distorsion being relatively weak, the cell symmetry was constrained to rhombohedral symmetry by using the averaged monoclinic cell parameters of the equilibrium structure. The different configurations were then relaxed at the equilibrium volume to conserve the rhombohedral symmetry.

Total energies were computed with the open-source code Quantum Espresso 
[35] using a GGA-based vdW-DF functional [36] which combines an optimized Becke86 exchange component with non-local van der Waals correlation part, as used in a previous work on He in boron carbide[25]. Defect calculations were carried out in a $2 \times 2 \times 2$ supercell to approach the dilute limit of the defect. The Brillouin zone of the supercell was sampled with a $2 \times 2 \times 2$ Monkhorst-Pack k-points mesh and the wave function kinetic energy cutoff was set to $80 \mathrm{Ry}$. In order to overcome the DFT band gap problem [37], calculations were also performed with the HSE06 hybrid functional [38, 39] to get a better description of charge transition levels of defects.

Charged defect calculations with the supercell method induce spurious interactions between the periodic images of the defect; thus long-range electrostatic interactions between charged defects were corrected with the Madelung monopole term of the Makov \& Paine approach[40, 41]. The latter is expressed, for a lattice of charges $q$ separated by a typical length $L$, as $\frac{q^{2} \alpha}{2 L \epsilon}$ where $\alpha$ stands for the Madelung constant of the lattice and $\epsilon$ stands for the dielectric constant of the material. Using the static dielectric constant calculated for the $\mathrm{B}_{11} \mathrm{C}^{\mathrm{p}} \mathrm{CBC}$ structure $\epsilon_{0} \approx 8$, the monopole correction was evaluated at $0.27 q^{2} \mathrm{eV}$. This first order correction was found sufficient to obtain converged energies within $0.05 \mathrm{eV}$ of the asymptotic value with our $2 \times 2 \times 2$ supercells, as shown in Fig. 2 .

Then the formation energy of a given defect in charge state $q$ is computed as :

$E_{f}^{q}\left(\mu_{e}\right)=E_{\text {tot }}^{\text {defect }}-E_{\text {tot }}^{\text {perfect }}+\sum_{i} \Delta n_{i} \mu_{i}^{*}+q \times\left(E_{V B M}+\mu_{e}\right)+E_{\text {corr }}^{\text {Madelung }}$

where $\Delta n_{i}$ and $\mu_{i}^{*}$ are associated respectively with the lack or excess of the atomic species $i$ and its chemical potential in the thermodynamic conditions of the material. In the following sections, formation energies are expressed in the carbon-rich thermodynamical condition. Carbon chemical potential was set to the atomic energy of graphite and helium chemical potential was set to the energy of an isolated He atom. The zero of the electron chemical potential was set to the valence band top of the perfect structure.

Eventually migration energies were calculated thanks to the Nudged Elastic Band method [42] as implemented in the PWneb module of Quantum Espresso distribution. Minimum energy migration paths were interpolated based on at least 5 discrete images, each relaxed below atomic forces of 0.05 $\mathrm{eV} / \AA$. 


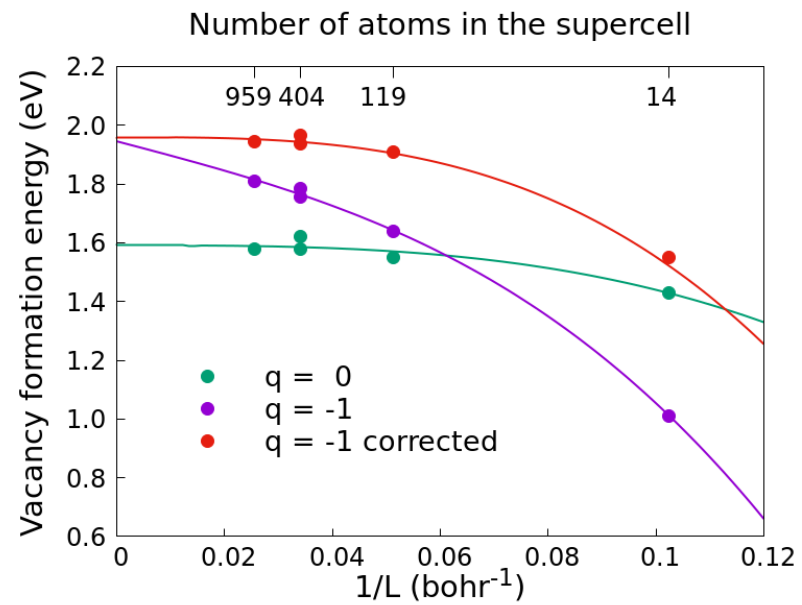

Figure 2: Convergence of the B1 vacancy formation energy versus supercell volume (number of atoms on the top $\mathrm{x}$-axis) for neutral and negative charge states with and without the monopole correction. The two points for the 404-atom supercell stands for $\Gamma$ and $2 \times 2 \times 2$ BZ sampling.

\section{Results}

In order to consider the interaction of vacancies with helium impurities and the possibility of vacancy-driven diffusion mechanisms, several key migrations are investigated. First we focus on the stability and migration of an isolated vacancy, a prerequisite to any mechanism involving vacancies. Then the association and dissociation of He impurities with vacancies, i.e., the formation and annihilation of substitutional impurities, are examined.

\subsection{Stability and mobility of an isolated vacancy}

For the sake of completeness, vacancy stability was studied on the 11 nonequivalent sites of the $\mathrm{B}_{11} \mathrm{C}^{\mathrm{p}} \mathrm{CBC}$ structure. Table 1 reports the formation energies of neutral vacancies and their charge transition levels.

Our charged vacancies calculations confirm that chain vacancies are more favorable than icosahedral vacancies [25, 26], especially the boron chain center vacancy which is the most stable vacancy in the structure by far.

Because of its predominance, the boron chain center (B1) vacancy stability was investigated using semilocal and hybrid functionals. The latter yielded slightly higher formation energies than GGA calculations but the 
qualitative picture is unchanged. In Fig. 3 the formation energies are represented with two different conventions:

In panel a) the GGA and HSE formation energies are compared with the usual x-axis convention, aligning at $\mu_{e}=0$ both valence band maxima, whereas in Fig. 3b GGA and HSE band structures are referred to a common absolute level[43] and only HSE valence band maximum is set to $\mu_{e}=0$. The experimental band gap at $2.09 \mathrm{eV}$ [44] is also displayed (as "Exp. CBM") to account for the discrepancies coming from the idealized $\mathrm{B}_{11} \mathrm{C}^{\mathrm{p}} \mathrm{CBC}$ structure of boron carbide. This double representation highlights the fact that the shift of the charge transition levels is mostly explained by the valence band shift between GGA and HSE calculations, as previously shown for a variety of defects in materials[43].

Based on our results, the chain center vacancy is expected to be positively charged or neutral in strongly or moderate $p$-type materials, and negatively charged in intrinsic and $n$-type conditions. The boron chain center vacancy adopts different geometries depending on its charge state. The distance between the two carbon atoms of the chain, evaluated at $2.90 \AA$ for the neutral defect with HSE functional, increases to $3.14 \AA$ when charged +1 and drops to $1.90 \AA$ when charged -1 . The negatively charged geometry change is similar to the formation of the $\mathrm{C}-\mathrm{C}$ bond described by Raucoules et al. [7] in high pressure LDA calculations. This is confirmed by the analysis of the charge density along the neutral and charged defective chains, as shown in Figure 4, with the significative increase of the electron density between the two carbon atoms in the negative charge state.

Icosahedral vacancies have formation energies ranging from 4.42 to 5.82 $\mathrm{eV}$ in the neutral state and they become positively charged near the valence band edge and negatively charged above $\mu_{e} \approx 1.0 \mathrm{eV}$ (see Table 1 ). Due to their very high formation energy, icosahedral vacancies are metastable configurations towards the chain center vacancy. Even though icosahedral vacancies have similar formation energies, polar and equatorial vacancies cannot be considered as equally stable. Indeed the relaxation starting from some of the equatorial configurations converged to the boron chain center configuration, showing that equatorial vacancies may be simply unstable. Probing the energy landscape around icosahedral vacancies confirmed this behavior showing that, for equatorial vacancies, the barrier to convert to a chain vacancy is either zero or extremely small and that the barrier between polar vacancies and the chain center vacancy is high enough to guarantee their stability ; thus in the following polar vacancies are considered stable 

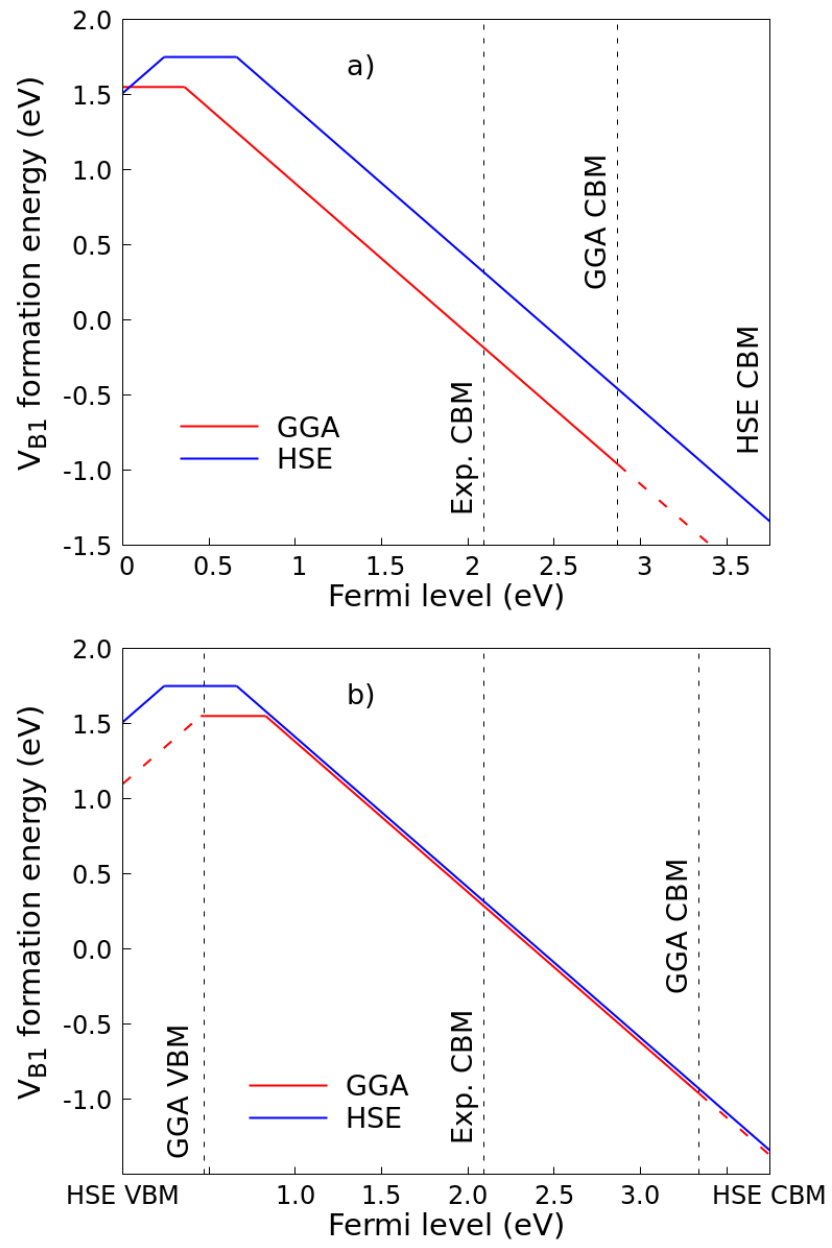

Figure 3: Formation energy of the chain center vacancy as a function of the Fermi level. Two different representations are displayed: a) GGA and HSE valence band maxima $(\mathrm{VBM})$ are aligned at $\left.\mu_{e}=0, \mathrm{~b}\right)$ HSE and GGA charge transition levels are referred to a common absolute level[43] and only HSE VBM is aligned at $\mu_{e}=0$. 


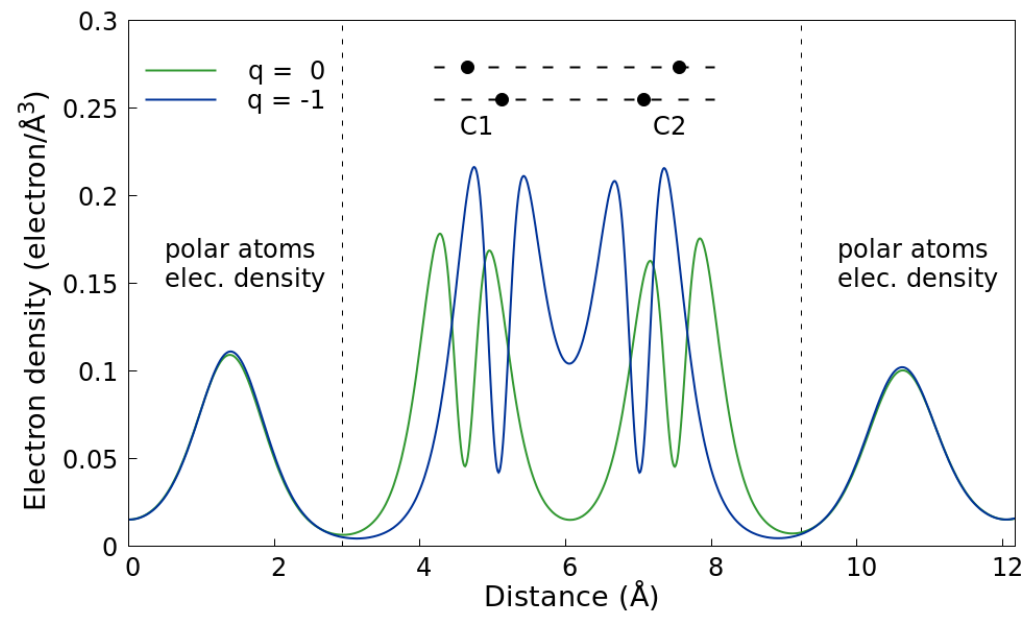

Figure 4: A comparison of the charge density along the line connecting the two carbon atoms $\mathrm{C}_{1}$ and $\mathrm{C}_{2}$ adjacent to the chain center boron vacancy in neutral and negative charge state. The black points above the curve indicate the positions of the carbon atoms in both configurations. The vertical dashed lines show the separation between icosahedra and chain regions. For the $\mathrm{Q}=+1$ charge state (not shown) the charge density is almost identical to the neutral case. 
and equatorial vacancies are considered unstable.

In order to limit the complexity of the study, carbon vacancies were not considered in the migration processes. Their migration would imply the creation of antisite defects whose study goes beyond the scope of this work. Moreover, equatorial vacancies being unstable, carbon chain vacancies would not have obvious migration paths. Therefore vacancy diffusion mechanisms are restricted to migrations involving polar boron vacancies and the chain center vacancy.

The B1 vacancy being the most stable configuration, elementary jumps between two chain centers were examined at first. The least energetic direct jump between two chain centers has been determined with an energy barrier of $4.73 \mathrm{eV}$ in the neutral state, which makes it very unlikely to be the main diffusion mechanism for vacancies. To find other migration paths for vacancies, polar vacancies are considered as intermediate configurations. The straightforward chain-polar mechanism combines a jump from the chain center to an icosahedra pole, possibly an exchange of the polar vacancy with one of its neighbour and eventually the landing of the vacancy on an other chain center (Fig. 5). These steps have respectively been evaluated with energy barriers of $3.72 \mathrm{eV}, 0.80 \mathrm{eV}$ and $0.85 \mathrm{eV}$. The limiting step is indeed the chain center to polar vacancy jump due to the large formation energy difference. Even if this chain-polar mechanism has a very high activation energy, it is still more favorable than the direct chain-chain migration path. Theoretically polar vacancies could also jump directly to other icosahedra with a calculated energy barrier of $1.99 \mathrm{eV}$ (in the neutral charge state) and provide long-range diffusion through a polar-polar mechanism with lower activation energy. The impact of this mechanism should be assessed as a function of vacancy supersaturation; however, at equilibrium, the contribution of this kind of mechanism is thought to be rather limited because the formation energy of polar vacancies is very high. The main diffusion mechanism of vacancies is thus the chain-polar mechanism, with a migration energy of $3.72 \mathrm{eV}$ and an activation energy (formation+migration) of $5.3 \mathrm{eV}$ for the neutral vacancy and that might be a few tenths of an eV lower for charged species, depending on the Fermi level position. This high activation energy for vacancy diffusion is not surprising if we compare it to other semiconducting carbides like silicon carbide.[45]

A recent work by You and coworkers[26], devoted to neutral defects, reached similar conclusions concerning migration energies. However, slightly higher energy barriers for the equatorial to chain center jump are reported, 


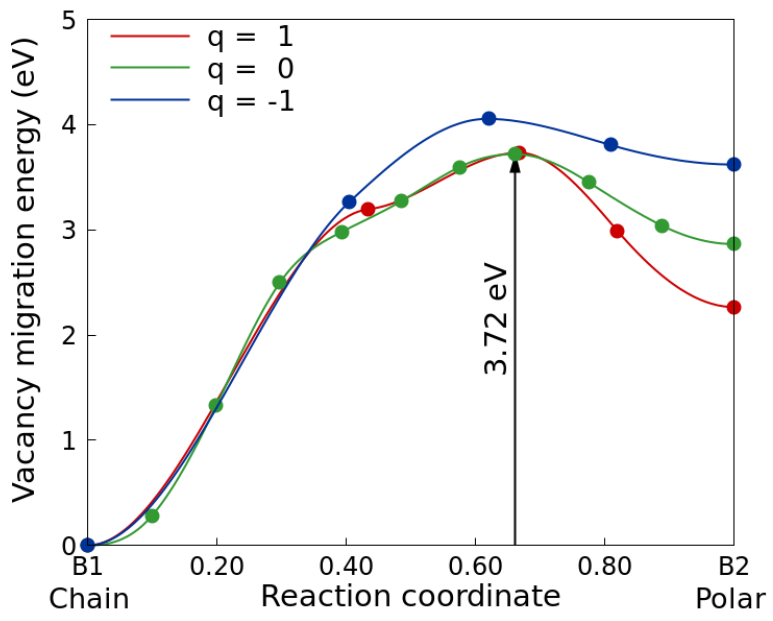

Figure 5: Migration energy profiles for the chain-to-polar conversion mechanism for vacancies in positive, neutral and negative charge state.

\begin{tabular}{c|ccccccc} 
& Site/Defect & $V^{0}$ & $\epsilon_{V}^{+1 / 0}$ & $\epsilon_{V}^{0 /-1}$ & $H e^{0}$ & $\epsilon_{H e}^{+1 / 0}$ & $\epsilon_{H e}^{0 /-1}$ \\
\hline chain center & B1 & 1.55 & - & 0.36 & 3.59 & 0.41 & 1.51 \\
\hline \multirow{2}{*}{ chain end } & C1 & 2.78 & - & 0.82 & $\times$ & $\times$ & $\times$ \\
& C2 & 2.92 & - & 0.80 & $\times$ & $\times$ & $\times$ \\
\hline \multirow{5}{*}{ polar } & C3 & 4.59 & 0.20 & 1.47 & 5.85 & 0.24 & 1.50 \\
& B2 & 4.42 & 0.58 & 1.11 & 5.77 & 0.59 & 1.33 \\
& B3 & 5.82 & - & 1.00 & 7.38 & 0.15 & 0.76 \\
& B4 & 5.36 & 0.52 & 0.96 & 6.71 & 0.57 & 1.16 \\
\hline \multirow{5}{*}{ equatorial } & B5 & 5.19 & 0.93 & 1.07 & $\times$ & $\times$ & $\times$ \\
& B6 & $\times$ & $\times$ & $\times$ & $\times$ & $\times$ & $\times$ \\
& B7 & $\times$ & $\times$ & $\times$ & $\times$ & $\times$ & $\times$ \\
& B8 & 4.49 & 0.28 & 0.90 & $\times$ & $\times$ & $\times$
\end{tabular}

Table 1: Formation energies and charge transition levels for vacancies and substitutional helium in boron carbide with the GGA functional; the $\times$ symbols indicate unstable configurations and the - symbols indicate charge transition levels lying outsite the band gap. Energy units are $\mathrm{eV}$. 
leading to consider the equatorial site as a stable position for migrations. In particular, the authors suggest that the migration from the polar to the chain center site is composed of two elementary jumps: one of $1.00 \mathrm{eV}$ between the polar and the equatorial site and another jump of $0.18 \mathrm{eV}$ between the equatorial and the chain center site. The migration from polar to chain center that we propose instead is a direct jump between the polar and the chain center site with an energy barrier around $0.80 \mathrm{eV}$. These discrepancies, which might arise from slightly different approximations (in particular the exchange-correlation functional) do not change the global picture of vacancy diffusion. Our study, in addition, clearly highlights the importance of the treatment of charged defects in boron carbide as illustrated with the stability of the chain center vacancy.

\subsection{Helium impurities and vacancies}

The introduction of vacancies in the model allows us to consider diffusion mechanisms other than the direct interstitial mechanism previously studied by Schneider et al.[25]. Indeed with the presence of intrinsic vacancies and interstitial impurities comes the possibility of substitutional impurity formation. Table 1 reports the formation energies and the charge transition levels of substitutional helium atoms and of vacancies.

Being chemically inert, the neutral substitutional helium adopts quite the same site preference as the neutral vacancy: the chain center helium is the most stable substitutional defect, the polar icosahedral helium is very expensive in terms of energy, and the equatorial helium is to be considered unstable. However, in the case of the substitutional impurity, the carbon chain sites are also unstable in all charge states.

Compared to the isolated vacancy, the charge transition levels of the chain center helium are shifted towards the middle of the band gap (Fig. 6a, 6b) while the polar sites charge transition levels are only marginally affected by the presence of helium. This behavior is presumably the consequence of the geometric nature of the chain center vacancy: the helium impurity is more favorable in an open chain and its presence prevents the formation of a $\mathrm{C}-\mathrm{C}$ bond.

To evaluate the effect of vacancies on the direct interstitial mechanism, the interactions between interstitial helium impurities and vacancies were studied. To remind the work of Schneider et al.[25], the two dominant types of helium interstitials sites are called "Type 2" and "Type 3" (or P2 and P3, see Fig. 7). The Type 2 position corresponds to 6 quasi-equivalent sites 

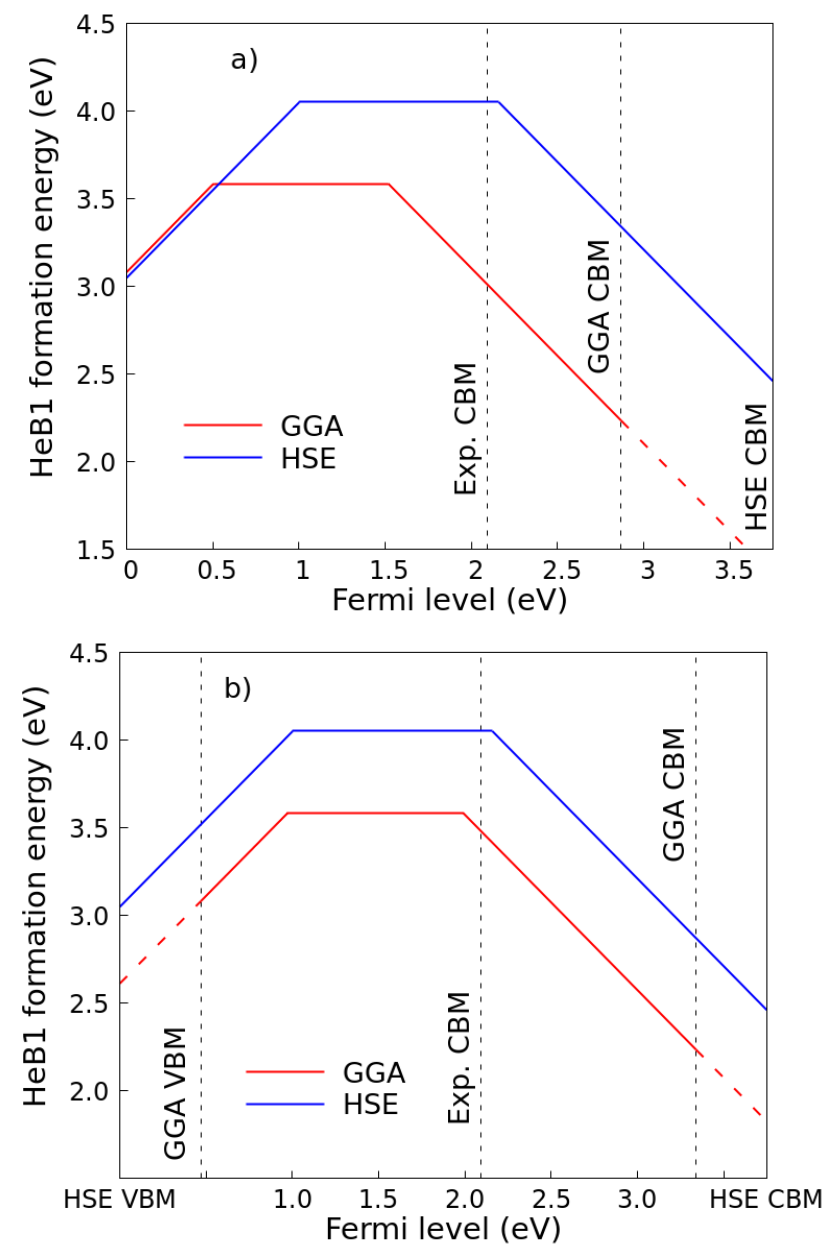

Figure 6: Formation energy of the chain center helium impurity as a function of the Fermi level. Two different representations are displayed: a) GGA and HSE VBM are aligned at $\left.\mu_{e}=0, \mathrm{~b}\right) \mathrm{HSE}$ and GGA charge transition levels are referred to a common absolute level[43] and only HSE VBM is aligned at $\mu_{e}=0$. 


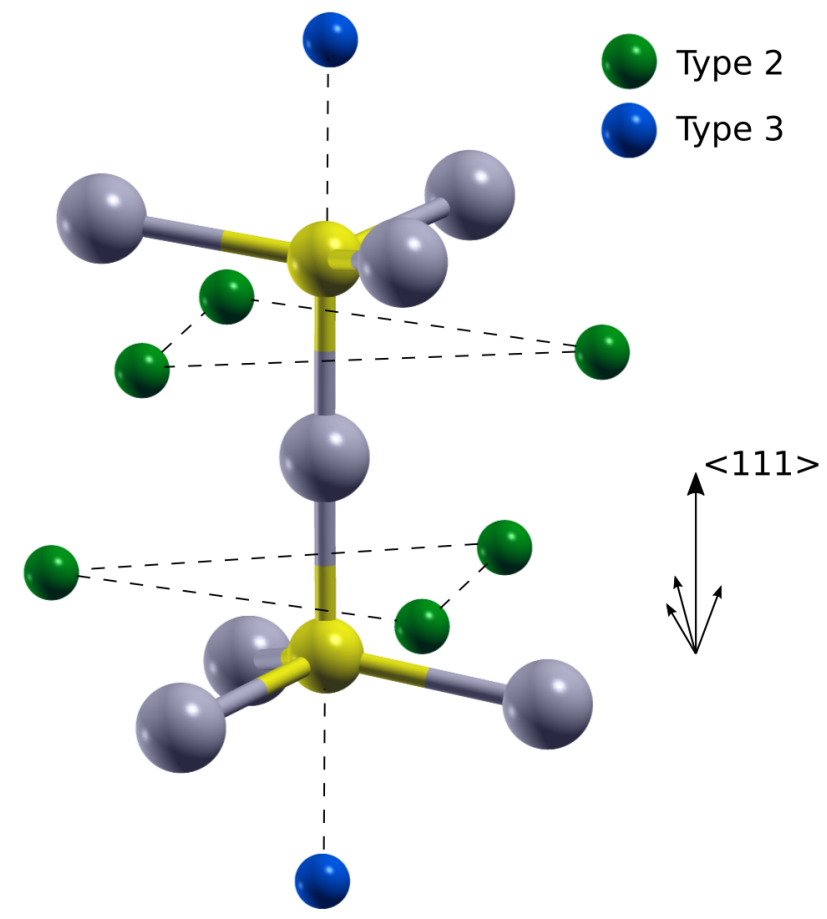

Figure 7: Helium interstitial positions in the rhombohedral elementary cell. Polar atoms are not represented for convenience. Type 2 and Type 3 helium sites are respectively coloured in green and blue.

located around the $\mathrm{C}-\mathrm{B}-\mathrm{C}$ chain. As for the Type 3 positions, it refers to two sites situated near the chain carbon atoms, in the prolongation of the $\mathrm{C}-\mathrm{B}-\mathrm{C}$ chain, along the $\langle 111\rangle$ direction of the rhombohedral cell. The formation energies of Type 2 and Type 3 sites are quite similar, ranging from 2.85 to $3.18 \mathrm{eV}$ with our parameters.

Our calculations show that the Type 2 interstitial configurations are unstable in presence of a positive and neutral B1 vacancy located nearby in the same $\{111\}$ slice (the region containing a CBC chain between two $\{111\}$ planes). In these charge states, the substitutional impurity is energetically more favorable and the migration barrier from Type 2 interstitial is less than $0.1 \mathrm{eV}$. However for the negative chain center vacancy, the substitutional helium is less favorable and the migration barrier reaches $0.98 \mathrm{eV}$ (Fig. 8c). Again these results are directly related to the geometric behavior of the B1 vacancy, which in the negative charge state forms a $\mathrm{C}-\mathrm{C}$ bond. Polar icosa- 
hedral vacancies are also strong attractors for interstitial helium atoms : adjacent Type 2 and 3 sites are no longer stable in presence of polar vacancies in any charge state. If we except the negative B1 substitutional atom, substitutional helium defects are favorable by more than $1.00 \mathrm{eV}$ compared to the interstitial helium defects in presence of vacancies.

As stated previously, in presence of substitutional impurities we can consider other diffusion mechanisms, in particular vacancy mechanisms and Frank-Turnbull dissociative ones [46, 47, 48].

Let us consider first classical vacancy mechanisms[47, 48]; they involve the exchange between a He substitutional impurity and a nearby vacancy, their association/dissociation as a bound complex, and the migration of the two members close or far from each others. A helium/vacancy exchange between two chain centers or a chain center and a polar site provided migration paths with energy barriers included between 0.60 and $1.60 \mathrm{eV}$. However, according to some test calculations we performed, the migration of vacancies in proximity of a substitutional helium atom involves barriers that are even higher than for the chain-polar mechanism described earlier in this paper for the isolated vacancy, whose activation energy is $3.72 \mathrm{eV}$. Thus, in spite of the relatively low exchange barriers, the very low mobility of vacancies compared to the mobility of interstitial helium atoms precludes the straightfoward identification of convenient vacancy-driven mechanisms.

Let us now discuss the Frank-Turnbull dissociative mechanism[46, 47, 48]; for this the main defect reactions to be considered are the association of a He interstitial with a vacancy to form a substitutional impurity and its dissociation. Given the high formation energies of the polar helium atoms we focus here only on the chain center vacancy: as a nearby Type 2 interstitial site is found to be unstable, the migration describing this mechanism involves the nearest Type 3 interstitial (Fig. 8a and 8c). For neutral and positive charge states, the migration energy from the interstitial to the substitutional configuration depends only on the Type 3 - Type 2 interstitial jump, whose energy barrier varies between 0.10 and $0.30 \mathrm{eV}$ [25]. For the negative charge state the migration energy required to form the substitutional impurity corresponds to the migration barrier between the Type 2 interstitial and the B1 site, i.e., $0.98 \mathrm{eV}$. As suggested above, this barrier is related to the energy necessary to break the $\mathrm{C}-\mathrm{C}$ bond formed on the $\mathrm{B} 1$ site.

The dissociation of the $\mathrm{B} 1$ substitutional helium into an interstitial-vacancy pair requires to overcome an energy barrier of 1.41, 1.05 and $0.76 \mathrm{eV}$ respectively for charge $+1,0$ and -1 . 


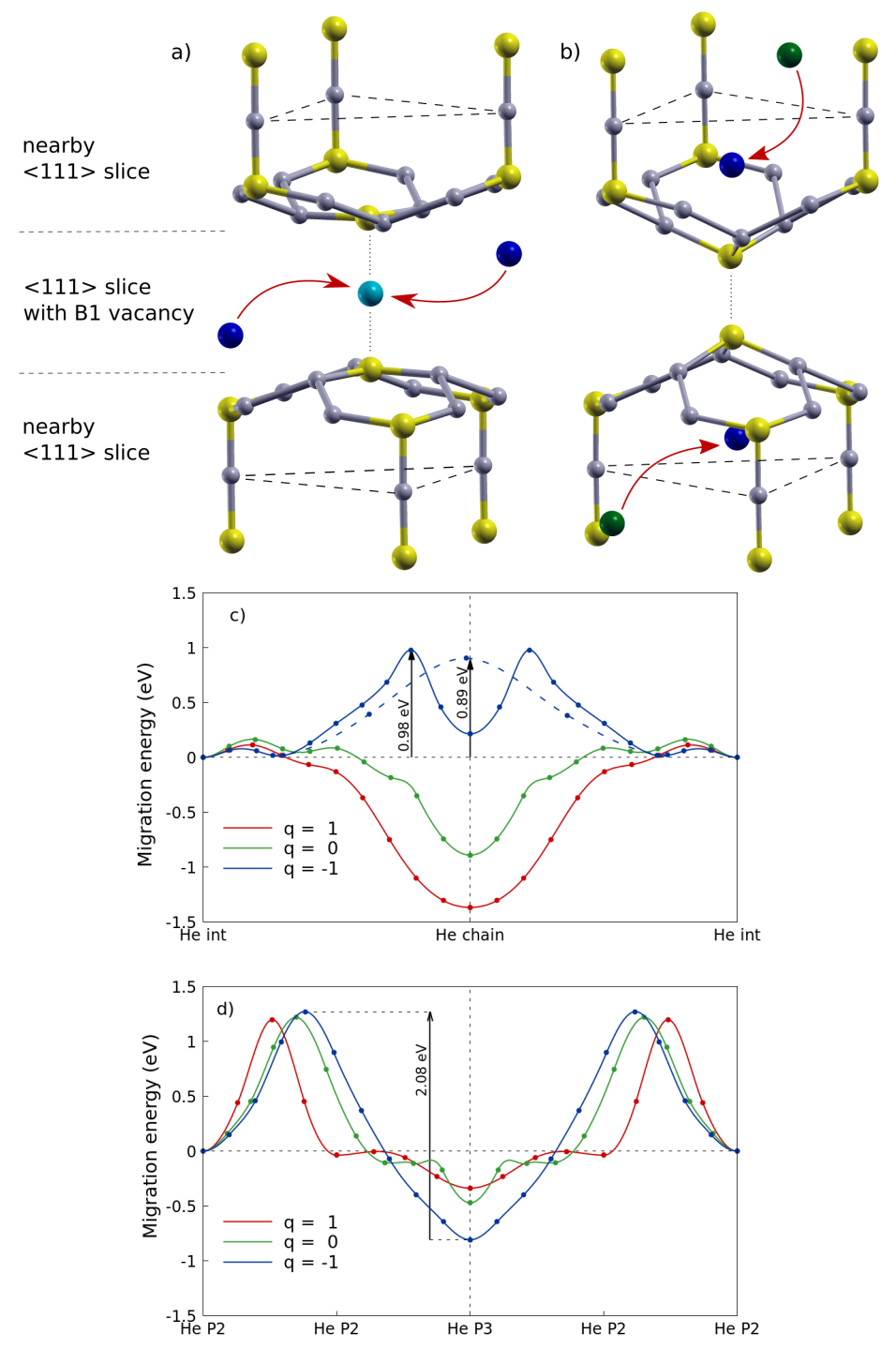

Figure 8: The two trapping mechanisms (some icosahedral atoms are not shown for readability): a) trapping with formation of chain-center helium substitutional; b) trapping on the Type 3 interstitial helium site. Migration barriers associated with these mechanisms are : c) Formation of chain center helium from Type 3 interstitial in three charge state (dashed line corresponds to the direct interstitial jump between two Type 2 positions); d) Type 2 - Type 3 migration with trapping from a vacancy located in an adjacent $\{111\}$ slice (stronger in negative charge state). 
From this dissociative mechanism on the chain center, two scenarios arise, depending of the charge state. The direct interstitial mechanism has a migration energy of $\approx 1.20 \mathrm{eV}$, which corresponds to the migration between two Type 2 interstitial helium atoms around the $\mathrm{C}-\mathrm{B}-\mathrm{C}$ chain (Fig. 9a).[25] Therefore in $p$-type conditions, when the vacancies are mostly neutral or positive, they clearly act as traps: in order to fully dissociate from the vacancy, a substitutional B1 helium has to cross the combined energy of the dissociation and Type 2 - Type 2 migration; an upper bound for the activation energy of this mechanism corresponds to the sum of the two steps, giving $\approx 2.3 \mathrm{eV}$ for the neutral state and $\approx 2.6 \mathrm{eV}$ for the positive state (Fig. 9b). On the other hand, in intrinsic or $n$-type conditions, the chain center vacancies are negative and even reduces locally the migration barrier. Indeed with the negative B1 vacancy, Type 2 interstitial positions are still stable and the Type 2 - Type 2 jump around the chain is still possible. This jump around the $\mathrm{C}-\mathrm{B}-\mathrm{C}$ chain has an energy barrier of $\approx 1.20 \mathrm{eV}$ as reminded before, but in the presence of the negative vacancy on the chain center, this energy barrier drops to $0.89 \mathrm{eV}$ (Fig. 8c). This means that the direct jump between two Type 2 interstitial positions is more likely than the formation of a B1 substitutional which has an energy barrier of $0.98 \mathrm{eV}$ in negative conditions for the B1 vacancies. Thus the direct interstitial mechanism would essentially be unaffected by negative vacancies, except from subtle correlation effects due to the alternation of slightly different barriers.

There is a further possibility that we have not yet considered : it is the trapping of an interstitial He by a vacancy without the formation of a substitutional He. Our calculations show that this can happen when an interstitial helium travels in a $\{111\}$ slice directly above or below a chain center vacancy (Fig. 8b). In particular, as shown in Fig. 8d, this trapping is stronger for a negative vacancy and only moderate for a neutral and positive one. This seems to be related both to a geometric and electrostatic effect, because in the negative charge state the defective chain is contracted forming a $\mathrm{C}-\mathrm{C}$ bond, leaving thus more space for the Type 3 interstitial. But also for the neutral vacancy, when the He passes through the Type 3 position, the defective chain shrinks to a distance of $1.76 \AA$, suggesting a charge transfer from the chain end to the chain center, and an attractive electrostatic interaction between the electron depleted He region and the electron augmented chain center; this is coherent with the previously observed correlation between charge depletion and He insertion[25].

The trapping tendency is now reversed with respect to the dissociative 
mechanism, where the strongest trap was the positive vacancy. Combining the two mechanisms - the dissociative and the interstitial trappingbasically we can observe trapping from vacancies of any charge state, with capture energies between 2.1 and $2.6 \mathrm{eV}$, and a slightly stronger effect in $p$-type conditions.

\section{Discussion}

The results presented in the previous section help us setting up a picture of the role of vacancies on He diffusion in boron carbide. To fix ideas let us recall that interstitial He diffusion, without vacancies, is expected to occur in $2 \mathrm{D}$ along $\{111\}$ slices with an activation energy of $1.2 \mathrm{eV}$ and in $3 \mathrm{D}$ with a higher activation energy of $2.2 \mathrm{eV}$. [25]

Most stable vacancies, i.e., chain center ones, can migrate, at best with an activation energy estimated at $3.7 \mathrm{eV}$ through a chain-polar mechanism. In case of high vacancy supersaturation, i.e., when the fraction of icosahedral (polar) vacancies represents a non-negligible concentration, a direct intericosahedral mechanism with $2 \mathrm{eV}$ activation energy has been found.

We consider two possible vacancy driven helium diffusion mechanisms [48]: the first, a usual vacancy mechanism, where He atoms, always in substitutional positions, exchange with vacancies; the second, a dissociative mechanism, where He is alternatively in substitutional and interstitial positions.

In the first case, although the exchange itself has low activation energy (0.6 eV at best), the migration energy of the isolated vacancy is very high $(3.7 \mathrm{eV})$; the migration of vacancies around He are not lower than that, and clearly high enough to exclude this mechanism to be the dominant one. However, when the concentration of vacancy is very high the mechanism involving only polar vacancy could be activated, with a migration energy of $2 \mathrm{eV}$; this is still higher than 2D interstitial diffusion, but slightly lower than $3 \mathrm{D}$ interstitial diffusion [25].

For the second case, the dissociative mechanism, we have to distinguish two further scenarios, depending on the Fermi level position. If the material is $p$-type, $\mathrm{B} 1$ vacancies are expected to be neutral or positive; in this case, the vacancy will trap helium in substitutional positions and the energy landscape will look like the one in Fig. 9b, where the activation energy, apart from subtle correlation effects between the He- $\mathrm{V}$ dissociation and He interstitial migration, is $2.3-2.6 \mathrm{eV}$. 

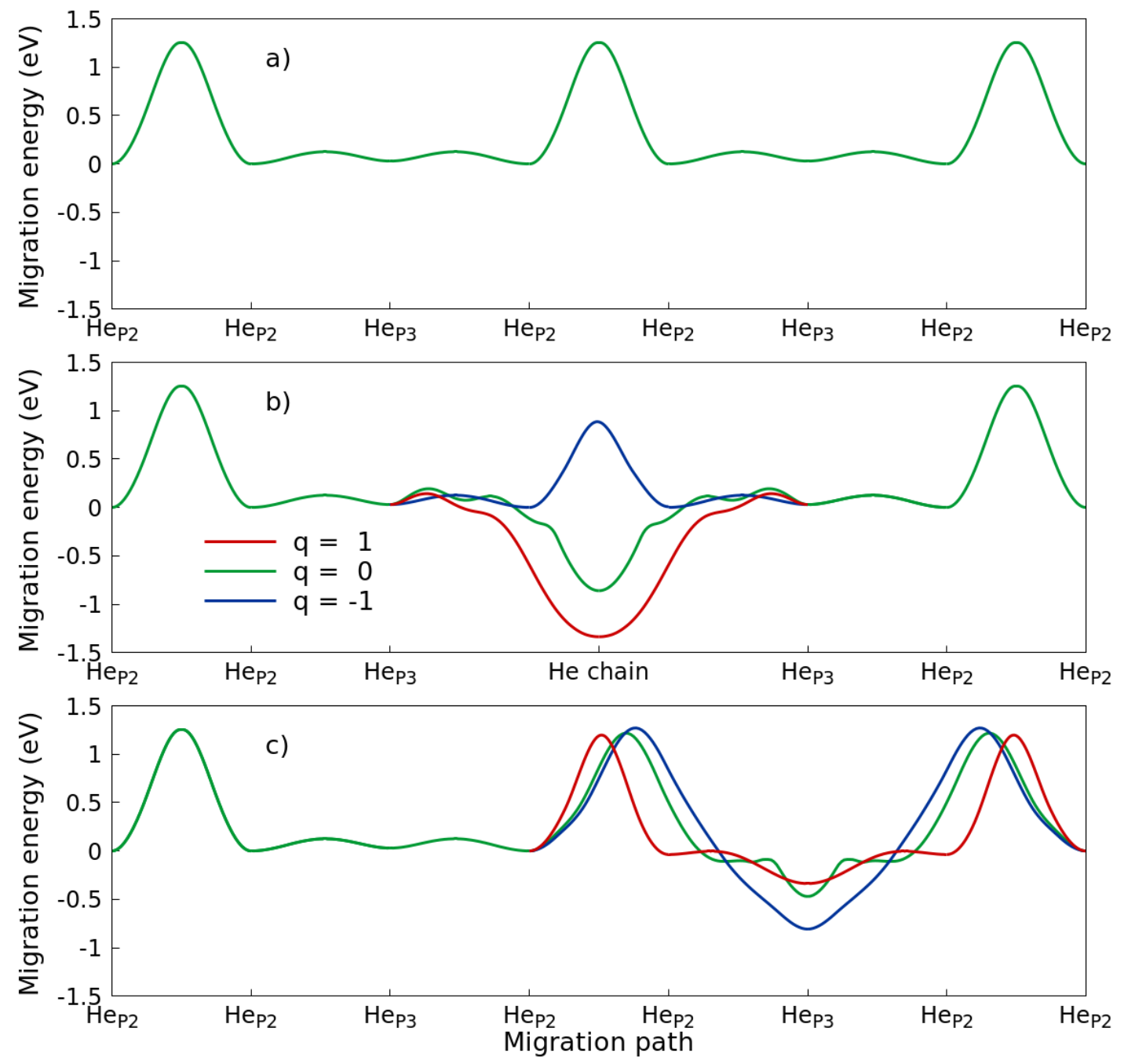

Figure 9: Migration energy profile for a neutral interstitial helium atom along a $\{111\}$ slice in boron carbide far from vacancies (a), encountering a chain center vacancy in the slice (b) or approaching a chain center vacancy in a neighboring $\{111\}$ slice (c). Three charge states for the vacancy are shown. 
If, conversely, the material is $n$-type, vacancies are expected to be in their negative state; in this case the dissociative mechanism is not any more relevant, because He interstitials would easily turn around vacancies in $\{111\}$ slices with an even slightly lower barrier $(0.89 \mathrm{eV}$, see Fig. 9b and dashed line in Fig. 8c). However, vacancies in nearby $\{111\}$ slices would trap He in interstitial form (Fig. 9c) and eventually produce a similar effect as the dissociative mechanism in $p$-type conditions, with an overall activation energy that, according to the concentration of vacancies, can reach $2.1 \mathrm{eV}$.

The results reported in this paper add a piece of information in order to interpret experiments where the mobility of He in irradiated materials is an important parameter. In a recent experimental work of one of us[49] $\mathrm{B}_{4} \mathrm{C}$ samples were implanted with He atoms, then annealed and He profiles were extracted through Nuclear Reaction Analysis (NRA). An activation energy of $E_{a}=2.03 \pm 0.18 \mathrm{eV}$ was obtained following a classic Arrhenius analysis for the intra-granular diffusion of He atoms in boron carbide.

Considering only interstitial mechanisms for helium diffusion, the threedimensional diffusion path with an activation energy of $2.2 \mathrm{eV}$ is in better agreement with the experimental value than the two-dimensional diffusion path with its activation energy of $1.2 \mathrm{eV}$. However, the measured profiles are supposed to integrate the $2 \mathrm{D}$ and $3 \mathrm{D}$ diffusion and, as such, to measure the lowest (2D) activation energy. In view of our results on the influence of vacancies we are keen to interpret the measured activation energy as a consequence of the presence of vacancies in the material, whose effect is to slow down the $2 \mathrm{D}$ diffusion to an activation energy of $\approx 2 \mathrm{eV}$. Vacancies are certainly present following He implantation in the measured sample. The profile of the damage peak almost coincides with the one of as-implanted He. This means that upon annealing, He can leave the damaged region because its mobility is in any case larger than that of vacancies. However, the vacancy concentration in the queues of the damage peak might be sufficient to influence He diffusion. Moreover, it has been suggested that antisites and vacancies and various forms of defective chains are difficult to avoid in boron carbide[50, 51], which strengthens the hypothesis that vacancies may influence He diffusion even outside of the damage peak.

We should nevertheless mention two further hypotheses excluding the role of vacancies : the first is that self-interstitial defects might diffuse faster than helium outside of the damaged region and provide other mechanisms to reduce He mobility. The second is that small (immobile) He bubbles (not visible with TEM) are formed during irradiation, and that the detrapping 
energy of He atoms from those bubbles might also affect the measured activation energy.

\section{Conclusion}

In summary, we presented here a first principle study of the stability and kinetics of vacancies and substitutional He impurities in various charge states in carbon-rich boron carbide $\left(\mathrm{B}_{4} \mathrm{C}\right)$. We showed that the most stable vacancy, the chain center boron one, is negatively charged in intrinsic and $n$-type conditions, and neutral or positive in $p$-type conditions. The charge state of the chain center vacancy clearly affects its topology: the positive and neutral vacancies correspond to wide open chains whereas the negative vacancy is better described by a shrinked chain with a $\mathrm{C}-\mathrm{C}$ bond. We also show that, independently of the charge state, the mobility of vacancies is very low $\left(\mathrm{E}_{m i g}>3 \mathrm{eV}\right)$ at equilibrium, with a possible enhancement $\left(\mathrm{E}_{\text {mig }} \simeq\right.$ $2 \mathrm{eV}$ ) in case of supersaturation of polar icosahedral boron vacancies.

It is found that substitutional He impurities are more favorable than interstitial helium in presence of vacancies in $p$-type conditions, when chain center vacancies are neutral or positively charged. The influence of vacancies on helium mobility is to slow down the 2D He diffusion along $\{111\}$ planes in two possible ways : in intrinsic and $n$-type conditions, when vacancies are negatively charged, 2D diffusion still occurs via an interstitial mechanism, with trapping of interstitial helium by vacancies located in a nearby $\{111\}$ slice. In $p$-type conditions, diffusion occurs via a dissociative mechanism, where He is occasionally trapped in vacancies as a substitutional impurity. In both cases the activation energy for detrapping is slightly higher $2 \mathrm{eV}$, which is compatible with very recents experimental results[49].

\section{Acknowledgements}

This work was granted access to the HPC resources of TGCC and CINES under the allocation 2017A0010906018 made by GENCI and under the allocation by CEA-DEN. We acknowledge partial financial support by the french research program NEEDS Matériaux.

\section{Data Statement}

The raw/processed data required to reproduce these findings cannot be shared at this time for legal reasons and because the data also forms part of 
an ongoing study.

\section{References}

[1] J. Rouault, P. Chellapandi, B. Raj, P. Dufour, C. Latge, L. Paret, P. L. Pinto, G. H. Rodriguez, G.-M. Gautier, G.-L. Fiorini, M. Pelletier, D. Gosset, S. Bourganel, G. Mignot, F. Varaine, B. Valentin, P. Masoni, P. Martin, J.-C. Queval, D. Broc, N. Devictor, in: D. G. Cacuci (Ed.), Handbook of Nuclear Engineering, volume 4, Springer, 2010, pp. 2321-2710. See section 5.3.2, pp. 2501-2506.

[2] D. Gosset, Structural Materials for Generation IV Nuclear Reactors, number 106 in Woodhead Publishing Series in Energy:, Elsevier Ltd., pp. $533-567$.

[3] S. Hayun, Bulletin of the American Ceramic Society 96 (2017) 20-26.

[4] A. U. Khan, V. Domnich, R. A. Haber, Bulletin of the American Ceramic Society 96 (2017) 30-35.

[5] D. Gosset, B. Kryger, J.-P. Bonal, C. Verdeau, K. Froment, Journal of Nuclear Materials 500 (2018) 166 - 175.

[6] J. E. Saal, S. Shang, Z.-K. Liu, Applied Physics Letters 91 (2007) 231915.

[7] R. Raucoules, N. Vast, E. Betranhandy, J. Sjakste, Phys. Rev. B 84 (2011) 014112.

[8] E. Betranhandy, N. Vast, J. Sjakste, Solid State Sciences 14 (2012) $1683-1687$.

[9] A. Ektarawong, S. I. Simak, L. Hultman, J. Birch, B. Alling, Phys. Rev. B 90 (2014) 024204.

[10] K. Rasim, R. Ramlau, A. Leithe-Jasper, T. Mori, U. Burkhardt, H. B. Borrmann, W. Schnelle, C. Carbogno, M. Scheffler, Y. Grin, Angewandte Chemie International Edition 130 (2018) 6238-6243.

[11] G. L. Copeland, R. G. Donnelly, W. R. Martin, Nuclear Technology 16 (1972) 226-237. 
[12] A. Jostsons, C. DuBose, G. Copeland, J. Stiegler, Journal of Nuclear Materials 49 (1973) 136-150.

[13] G. W. Hollenberg, B. Mastel, J. A. Basmajian, Journal of the American Ceramic Society 63 (1980) 376-380.

[14] V. Saraswati, G. V. N. Rao, J. Mater. Sci. Lett. 4 (1985) 260.

[15] V. I. Shcherbak, V. P. Tarasikov, V. N. Bykov, V. A. Rudenko, Soviet Atomic Energy 60 (1986) 227-230.

[16] T. Stoto, J. Ardonceau, L. Zuppiroli, M. Castiglioni, B. Weckermann, Radiation Effects 105 (1987) 17-30.

[17] F. Zhang, X. Wang, J. B. Wierschke, L. Wang, Scripta Materialia 109 (2015) $28-33$.

[18] T. Marumaya, S. Onose, T. Kaito, H. Horiuchi, Journal of Nuclear Science and Technology 34 (1997) 1006-1014.

[19] D. Simeone, C. Mallet, P. Dubuisson, G. Baldinozzi, C. Gervais, J. Maquet, Journal of Nuclear Materials 277 (2000) 1-10.

[20] V. Motte, D. Gosset, S. Miro, S. Doriot, S. Surblé, N. Moncoffre, EPJ Nuclear Sci. Technol. 1 (2015) 16.

[21] Y. You, K. Yoshida, T. Inoue, T. Yano, Journal of Nuclear Science and Technology (2018) 1-9.

[22] J. C. Clayton, W. A. Bostrom, F. C. Schrag, The release of helium from slightly irradiated boron carbide and boron carbide-silicon carbide plates, Technical Report WAPD-255, Bettis Atomic Power Laboratory, Pittsburgh, PA. operated for the U.S. Atomic Energy Commission by Westinghouse Electric Corporation,, Washington, D.C., 1962.

[23] V. V. Svetukhin, D. N. Suslov, V. D. Risovanyi, P. F. Salikh-zade, Atomic Energy 98 (2005) 177.

[24] C. E. Beyer, G. W. Hollenberg., S. A. Duran, A physically based model for helium release from irradiated boron carbide, Technical Report HEDL-SA-1833-FP, Hanford Engineering Development Laboratory, 1979. 
[25] A. Schneider, G. Roma, J.-P. Crocombette, V. Motte, D. Gosset, Journal of Nuclear Materials 496 (2017) 157-162.

[26] Y. You, K. Yoshida, T. Yano, Jpn. J. Appl. Phys., Part 157 (2018) 055801.

[27] M. Bouchacourt, F. Thevenot, Journal of the Less Common Metals 67 (1979) 327-331.

[28] K. A. Schwetz, P. Karduck, Journal of the Less Common Metals 175 (1991) $1-11$.

[29] S. V. Konovalikhin, V. I. Ponomarev, Russian Journal of Physical Chemistry A 84 (2010) 1445-1448.

[30] H. Werheit, S. Shalamberidze, Journal of Physics: Condensed Matter 24 (2012) 385406.

[31] R. Lazzari, N. Vast, J. M. Besson, S. Baroni, A. D. Corso, Physical Review Letters 83 (1999) 3230-3233.

[32] N. Vast, R. Lazzari, J. Besson, S. Baroni, A. D. Corso, Computational Materials Science 17 (2000) 127-132.

[33] F. Mauri, N. Vast, C. J. Pickard, Physical Review Letters 87 (2001).

[34] H. K. Clark, J. L. Hoard, Journal of the American Chemical Society 65 (1943) 2115-2119.

[35] P. Giannozzi, O. Andreussi, T. Brumme, O. Bunau, M. B. Nardelli, M. Calandra, R. Car, C. Cavazzoni, D. Ceresoli, M. Cococcioni, N. Colonna, I. Carnimeo, A. D. Corso, S. de Gironcoli, P. Delugas, R. A. DiStasio, A. Ferretti, A. Floris, G. Fratesi, G. Fugallo, R. Gebauer, U. Gerstmann, F. Giustino, T. Gorni, J. Jia, M. Kawamura, H.-Y. Ko, A. Kokalj, E. Kçkbenli, M. Lazzeri, M. Marsili, N. Marzari, F. Mauri, N. L. Nguyen, H.-V. Nguyen, A. O. de-la Roza, L. Paulatto, S. Poncé, D. Rocca, R. Sabatini, B. Santra, M. Schlipf, A. P. Seitsonen, A. Smogunov, I. Timrov, T. Thonhauser, P. Umari, N. Vast, X. Wu, S. Baroni, Journal of Physics: Condensed Matter 29 (2017) 465901.

[36] J. Klimeš, D. R. Bowler, A. Michaelides, Phys. Rev. B 83 (2011) 195131. 
[37] J. P. Perdew, International Journal of Quantum Chemistry 28 (2009) 497-523.

[38] J. Heyd, G. E. Scuseria, M. Ernzerhof, The Journal of Chemical Physics 118 (2003) 8207-8215.

[39] J. Heyd, G. E. Scuseria, M. Ernzerhof, J. Chem. Phys. 124 (2006) 219906.

[40] M. Leslie, N. J. Gillan, Journal of Physics C: Solid State Physics 18 (1985) 973-982.

[41] G. Makov, M. C. Payne, Physical Review B 51 (1995) 4014-4022.

[42] G. Henkelman, B. P. Uberuaga, H. Jónsson, The Journal of Chemical Physics 113 (2000) 9901-9904.

[43] A. Alkauskas, P. Broqvist, A. Pasquarello, Phys. Rev. Lett. 101 (2008) 046405.

[44] H. Werheit, M. Laux, U. Kuhlmann, R. Telle, physica status solidi (b) 172 (1992) K81-K86.

[45] O. N. Bedoya-Martínez, G. Roma, Phys. Rev. B 82 (2010) 134115.

[46] F. C. Frank, D. Turnbull, Phys. Rev. 104 (1956) 617-618.

[47] J. L. Bocquet, G. Brebec, Y. Limoge, Diffusion in Metals and Alloys, Elsevier Science BV, pp. 535-668.

[48] H. Bracht, Phys. Rev. B 75 (2007) 035210.

[49] V. Motte, D. Gosset, J. Nucl. Mater. (2018). Submitted.

[50] G. H. Kwei, B. Morosin, The Journal of Physical Chemistry 100 (1996) 8031-8039.

[51] R. Schmechel, H. Werheit, Journal of Solid State Chemistry 154 (2000) $61-67$. 